\title{
Enantio- and diastereoselective allylmetallations: an easy and efficient access to the $A B$ spiroketal of spongistatin
}

\author{
Florent Allais and Janine Cossy* \\ Laboratoire de Chimie Organique, associé au CNRS, \\ ESPCI, 10 rue Vauquelin, F-75231 Paris Cedex 05, France \\ Janine.cossy@espci.fr
}

\section{Supporting information}

\section{General :}

TLC was performed on Merck $60 \mathrm{~F}_{254}$ silica gel plates and visualized either with a UV lamp $(254 \mathrm{~nm})$, or by using a solution of $p$-anisaldehyde/sulfuric acid/acetic acid in EtOH followed by heating. Flash chromatography was performed with Merck Geduran Si60 silica gel (40-63 $\mathrm{UM}$ ) with petroleum ether (PE) and ethyl acetate (AcOEt). Infrared (IR) spectra were recorded on a Perkin-Elmer 298, wave-numbers are indicated in $\mathrm{cm}^{-1} .{ }^{1} \mathrm{H}$ NMR spectra were recorded on a Bruker AC 400 at $400 \mathrm{MHz}$ and data are reported as follows: chemical shift in ppm from tetramethylsilane as an internal standard $\left(\mathrm{CDCl}_{3} \delta 7.27 \mathrm{ppm}\right)$, multiplicity (s = singlet, $\mathrm{d}=$ doublet, $\mathrm{t}=$ triplet, $\mathrm{q}=$ quartet, $\mathrm{m}=$ multiplet or overlap of non equivalent resonances), integration. ${ }^{13} \mathrm{C}$ NMR spectra were recorded on a Bruker AC 400 at $125 \mathrm{MHz}$ and data are reported as follows: chemical shift in ppm from tetramethylsilane with the solvent as an internal indicator $\left(\mathrm{CDCl}_{3} \delta 77.0 \mathrm{ppm}\right)$, multiplicity with respect to proton (deduced from DEPT experiments, $\mathrm{s}=$ quaternary $\mathrm{C}, \mathrm{d}=\mathrm{CH}, \mathrm{t}=\mathrm{CH}_{2}, \mathrm{q}=\mathrm{CH}_{3}$ ). Mass spectra with electronic impact (MS-EI) were recorded from a Hewlett-Packard tandem 5890A GC (12 m capillary column $)-5971 \mathrm{MS}(70 \mathrm{eV})$.

\section{(S)-2-(2-Benzyloxy-ethyl)-oxirane (1)}

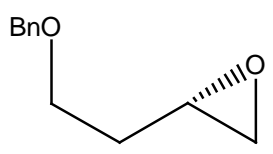

In a round-bottom flask under Ar, the $(S)$-4-benzyloxy-1,2-butanediol (4.20 g, $21.4 \mathrm{mmol}$, 1 equiv) was dissolved in $\mathrm{CHCl}_{3}(50 \mathrm{~mL})$ and pyridine $(3.6 \mathrm{~mL})$ and the resultant mixture was stirred at $0{ }^{\circ} \mathrm{C}$ for $5 \mathrm{~min}$. After this time, $p$-toluenesulfonyl chloride $(4.10 \mathrm{~g}, 21.4 \mathrm{mmol}$, 1 equiv) was added in $\mathrm{CHCl}_{3}(5 \mathrm{~mL})$ over a period of $2 \mathrm{~min}$. The mixture was stirred at $0{ }^{\circ} \mathrm{C}$ for $2.5 \mathrm{~h}$, after which time the cooling bath was removed and the mixture was stirred for an additional $2 \mathrm{~h}$ at $\mathrm{rt} \mathrm{Et}_{2} \mathrm{O}(200 \mathrm{~mL})$ was then added with stirring and the mixture was washed with water $(2 \times 15 \mathrm{~mL})$. the organic layer was dried over anhydrous $\mathrm{MgSO}_{4}$, filtered, and concentrated in vacuo to give the desired material $(7.00 \mathrm{~g}, 94 \%)$, which exhibited a major spot on TLC $\left(\mathrm{R}_{\mathrm{f}} 0.80\right.$, hexane/AcOEt 2:1) with a trace of starting material. The crude tosylate was used in the next reaction without further purification due to its extreme reactivity.

Tosylate $(3.50 \mathrm{~g}, 10.0 \mathrm{mmol}, 1$ equiv) was dissolved in dry $\mathrm{MeOH}(30 \mathrm{~mL})$ and cooled to $-10{ }^{\circ} \mathrm{C}$, and anhydrous potassium carbonate $(1.70 \mathrm{~g}, 12.3 \mathrm{mmol}, 1.2 \mathrm{equiv})$ was added. The reaction mixture was stirred at $-10{ }^{\circ} \mathrm{C}$ for $3 \mathrm{~h}$ and allowed to warm to $\mathrm{rt}$ with stirring for an 
extra $2 \mathrm{~h}$. After this time $\mathrm{Et}_{2} \mathrm{O}(50 \mathrm{~mL})$ was added and the resulting precipitate was filtered over a pad of silica gel. The precipitate was washed with $\mathrm{Et}_{2} \mathrm{O}(100 \mathrm{~mL})$ and the combined filtrates were concentrated in vacuo. The residue was purified by flash chromatography (PE/ ACOEt : 9/1) and afforded the pure epoxide 1 (1.65 g, 98\%).

${ }^{1} \mathrm{H}$ NMR (400 MHZ, $\left.\mathrm{CDCl}_{3}\right): \delta 7.38$ (bs, 5H), $4.58(\mathrm{~s}, 2 \mathrm{H}), 3.62(\mathrm{~m}, 2 \mathrm{H}), 3.10(\mathrm{~m}, 1 \mathrm{H}), 2.76$ $(\mathrm{m}, 1 \mathrm{H}), 2.51(\mathrm{~m}, 1 \mathrm{H}), 1.85(\mathrm{~m}, 2 \mathrm{H})$

${ }^{13} \mathrm{C}$ NMR (125 MHz, $\left.\mathrm{CDCl}_{3}\right): \delta 138.4(\mathrm{~s}), 128.4,128.0,127.8(3 \mathrm{~d}), 731 .(\mathrm{t}), 67.1(\mathrm{t}), 50.0(\mathrm{~d})$, $47.0(\mathrm{t}), 33.0(\mathrm{t})$

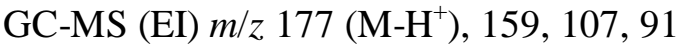

IR (film) v 1092, 908, $730 \mathrm{~cm}^{-1}$

$[\alpha]_{25}^{\mathrm{D}}=-12.1^{\circ}\left(c=0.3, \mathrm{CHCl}_{3}\right)\left[\right.$ lit. values $\left.{ }^{1}[\alpha]^{\mathrm{D}}{ }_{19}=-14.8^{\circ}(c=0.8, \mathrm{EtOH})\right]$

(3R)-1-Benzyloxy-5-methyl-hex-5-en-3-ol (2)

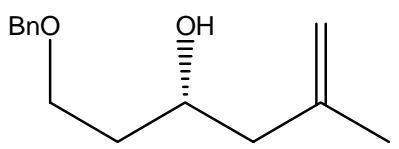

To a flame-dried round-bottom flask was added $\mathrm{CuI}(0.107 \mathrm{~g}, 0.56 \mathrm{mmol}, 0.1$ equiv) under Ar. Freshly distilled $\mathrm{Et}_{2} \mathrm{O}(20 \mathrm{~mL})$ was added and the suspension was cooled to $-78{ }^{\circ} \mathrm{C}$. Isopropenylmagnesium bromide $0.5 \mathrm{M}$ solution $(22.4 \mathrm{~mL}, 11.2 \mathrm{mmol}, 2$ equiv) was added dropwise and the mixture was allowed to stir at this temperature for $30 \mathrm{~min}$, then warmed to $40{ }^{\circ} \mathrm{C}$ for $10 \mathrm{~min}$. After cooling to $-78{ }^{\circ} \mathrm{C}$, a solution of $\mathbf{1}\left(1.0 \mathrm{~g}, 5.6 \mathrm{mmol}, 1\right.$ equiv) in $\mathrm{Et}_{2} \mathrm{O}$ $(10 \mathrm{~mL})$ was added and the resulting mixture was stirred for $1 \mathrm{~h}$. The solution was quenched by addition of a saturated aqueous $\mathrm{NH}_{4} \mathrm{Cl}$ solution $(10 \mathrm{~mL})$ and extracted with $\mathrm{CH}_{2} \mathrm{Cl}_{2}(3 \mathrm{x}$ $50 \mathrm{~mL}$ ). The combined organic layers were dried over anhydrous $\mathrm{MgSO}_{4}$, filtered and concentrated in vacuo. The resulting oil was purified by flash chromatography $(6: 4$ PE/AcOEt) and afforded pure 2 as a clear oil (11.59 g, 94\%).

${ }^{1} \mathrm{H} \mathrm{NMR}\left(400 \mathrm{MHZ}, \mathrm{CDCl}_{3}\right): \delta 7.31(\mathrm{~m}, 5 \mathrm{H}), 4.83(\mathrm{~s}, 1 \mathrm{H}), 4.76(\mathrm{~s}, 1 \mathrm{H}), 4.52(\mathrm{~s}, 2 \mathrm{H}), 3.96(\mathrm{~m}$, $1 \mathrm{H}), 3.67(\mathrm{~m}, 2 \mathrm{H}), 2.77(\mathrm{~d}, J=2.5 \mathrm{~Hz}, \mathrm{OH}), 2.18(\mathrm{~m}, 2 \mathrm{H}), 1.76(\mathrm{~m}, 2 \mathrm{H}), 1.75(\mathrm{~s}, 3 \mathrm{H})$

${ }^{13} \mathrm{C} \mathrm{NMR}\left(125 \mathrm{MHz}, \mathrm{CDCl}_{3}\right): \delta 142.8(\mathrm{~s}), 138.1(\mathrm{~s}), 128.5,127.9,127.7$ (3d), 113.1 (t), 73.3 $(\mathrm{t}), 68.7(\mathrm{t}), 68.2(\mathrm{~d}), 46.1(\mathrm{t}), 36.3(\mathrm{t}), 22.6(\mathrm{q})$

GC-MS (EI) m/z $219\left(\mathrm{M}-\mathrm{H}^{+}\right), 165,107,91$

IR (film) v 1645, 1100, 1080, 892, 740, $700 \mathrm{~cm}^{-1}$

$[\alpha]_{25}^{\mathrm{D}}=-3.39^{\circ}\left(c=0.50, \mathrm{CHCl}_{3}\right)\left[\right.$ lit. values $\left.{ }^{2}[\alpha]_{20}^{\mathrm{D}}=-3.75^{\circ}\left(c=2.49, \mathrm{CHCl}_{3}\right)\right]$ 
(4S)-6-Benzyloxy-4-hydroxy-hexan-2-one (3)

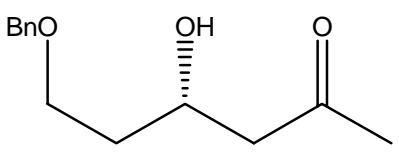

To a solution of 2 ( $10 \mathrm{~g}, 45 \mathrm{mmol}, 1$ equiv) in a 3:1 dioxane $/ \mathrm{H}_{2} \mathrm{O}$ mixture ( $\left.385 \mathrm{~mL}: 125 \mathrm{~mL}\right)$ at $\mathrm{rt}$ was successively added $\mathrm{OsO}_{4}(0.9 \mathrm{mmol}, 0.02$ equiv $), 2,6$-lutidine $(11.54 \mathrm{~mL}, 100 \mathrm{mmol}$, 2 equiv) and $\mathrm{NaIO}_{4}(21.34 \mathrm{~g}, 200 \mathrm{mmol}, 4$ equiv). The solution was stirred at this temperature until completion $\left(\mathrm{ca} .3 \mathrm{~h}\right.$ ) then quenched by addition of water $(200 \mathrm{~mL})$ followed by $\mathrm{CH}_{2} \mathrm{Cl}_{2}$ (300 mL). The aqueous layer was extracted with $\mathrm{CH}_{2} \mathrm{Cl}_{2}(3 \times 200 \mathrm{~mL})$, the combined organic layers were dried over anhydrous $\mathrm{MgSO}_{4}$, filtered and concentrated in vacuo. The residue was purified by flash chromatography (PE/AcOEt 8:2) to afford the pure hydroxyketone 3 (9.39 g, $94 \%)$.

${ }^{1} \mathrm{H}$ NMR (400 MHZ, $\left.\mathrm{CDCl}_{3}\right): \delta 7.31(\mathrm{~m}, 5 \mathrm{H}), 4.48(\mathrm{~s}, 2 \mathrm{H}), 4.23(\mathrm{~m}, 1 \mathrm{H}), 3.62(\mathrm{~m}, 2 \mathrm{H}), 3.49$ (bs, $1 \mathrm{OH}), 2.58(\mathrm{~m}, 2 \mathrm{H}), 2.13(\mathrm{~s}, 3 \mathrm{H}), 1.74(\mathrm{~m}, 2 \mathrm{H})$.

${ }^{13} \mathrm{C}$ NMR (125 MHz, $\left.\mathrm{CDCl}_{3}\right): \delta 209.1(\mathrm{~s}), 138.1(\mathrm{~s}), 128.4,127.9,127.7(3 \mathrm{~d}), 73.2(\mathrm{t}), 67.8$ $(\mathrm{t}), 66.3(\mathrm{~d}), 50.3(\mathrm{t}), 36.2(\mathrm{t}), 30.8(\mathrm{q})$

GC-MS (EI) $m / z 222\left(\mathbf{M}^{+}\right), 177,149,76$

IR (film) v 3435, 2862, 1709, 1358, 1274, 1026, $698 \mathrm{~cm}^{-1}$

$[\alpha]_{20}^{\mathrm{D}}=+12.0^{\circ}\left(c=0.05, \mathrm{CHCl}_{3}\right)$

(3S,5R)-1-Benzyloxy-5-methyl-oct-7-ene-3,5-diol (4)

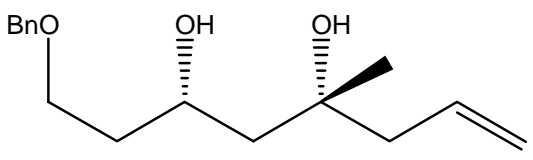

To a flame-dried round-bottom flask under Ar was added $\mathrm{CH}_{2} \mathrm{Cl}_{2}(27 \mathrm{~mL}), \mathrm{SnCl}_{4}(1.27 \mathrm{~mL}$, $10.9 \mathrm{mmol}, 1$ equiv) and allyltrimethylsilane (1.73 $\mathrm{mL}, 10.9 \mathrm{mmol}, 1$ equiv) and the solution was stirred at $\mathrm{rt}$ for $12 \mathrm{~h}$. The latter solution was transferred by a canula to a round-bottom flask containing a solution of $\beta$-hydroxyl-ketone $3\left(2.0 \mathrm{~g}, 9.08 \mathrm{mmol}, 0.8\right.$ equiv) in $\mathrm{CH}_{2} \mathrm{Cl}_{2}$ $(10 \mathrm{~mL})$ at $-78{ }^{\circ} \mathrm{C}$. The mixture was allowed to stir at this temperature for $1 \mathrm{~h}$. Work-up entailed addition of $\mathrm{MeOH}(2 \mathrm{~mL})$, saturated aqueous $\mathrm{NH}_{4} \mathrm{Cl}$ solution $(5 \mathrm{~mL})$ and extraction of the resulting mixture with $\mathrm{CH}_{2} \mathrm{Cl}_{2}(3 \times 20 \mathrm{~mL})$. The combined organic layers were dried over anhydrous $\mathrm{MgSO}_{4}$, filtered and concentrated in vacuo. The residue was purified by flash chromatography (PE/AcOEt 8:2) to afford the pure diol 4 (1.85 g, 77\%).

${ }^{1} \mathrm{H}$ NMR (400 MHZ, $\left.\mathrm{CDCl}_{3}\right): \delta 7.32(\mathrm{~m}, 5 \mathrm{H}), 5.87(\mathrm{ddt}, J=7.4,10.2,17.1 \mathrm{~Hz}, 1 \mathrm{H}), 5.10(\mathrm{~m}$, $2 \mathrm{H}), 4.51(\mathrm{~s}, 2 \mathrm{H}), 4.24(\mathrm{ddt}, J=2.2,2.6,8.4 \mathrm{~Hz}, 1 \mathrm{H}), 3.83(\mathrm{bs}, 1 \mathrm{OH}), 3.69(\mathrm{~m}, 2 \mathrm{H}), 3.57$ (bs, $1 \mathrm{OH}), 2.23(\mathrm{~d}, J=7.4 \mathrm{~Hz}), 2 \mathrm{H}), 1.86-1.1 .62(\mathrm{~m}, 2 \mathrm{H}), 1.64(\mathrm{~d}, J=12.2 \mathrm{~Hz}, 1 \mathrm{H}), 1.43(\mathrm{dd}, J=$ 2.2, $12.2 \mathrm{~Hz}, 1 \mathrm{H}), 1.28(\mathrm{~s}, 3 \mathrm{H})$.

${ }^{13} \mathrm{C}$ NMR (125 MHz, $\left.\mathrm{CDCl}_{3}\right): \delta 137.8(\mathrm{~s}), 134.1(\mathrm{~d}), 128.5,127.8,127.7(3 \mathrm{~d}), 118.3(\mathrm{t}), 73.4$ $(\mathrm{t}), 72.5(\mathrm{~s}), 69.2(\mathrm{~d}), 68.9(\mathrm{t}), 48.7(\mathrm{t}), 45.9(\mathrm{t}), 37.2(\mathrm{t}), 25.9(\mathrm{q})$ 
GC-MS (EI) m/z $263\left(\mathrm{M}-\mathrm{H}^{+}\right), 249,107,91$

IR (film) v 3376, 2919, $1098 \mathrm{~cm}^{-1}$

$[\alpha]_{20}^{\mathrm{D}}=-4.53^{\circ}\left(c=0.05, \mathrm{CHCl}_{3}\right)$

(4-Allyl-6-(2-benzyloxy-ethyl)-2,2,4-trimethyl-[1,3]dioxane (5)

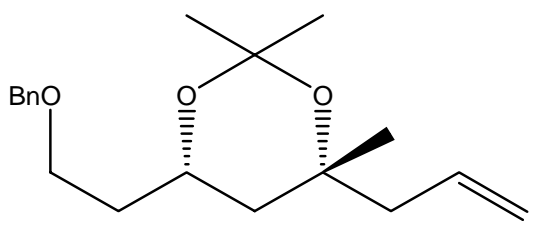

Compound 4 (1.0 g, $3.8 \mathrm{mmol}, 1$ equiv) was dissolved in $\mathrm{CH}_{2} \mathrm{Cl}_{2}$ (8 mL) at rt. 2,2dimethoxypropane ( $4.6 \mathrm{~mL}, 38 \mathrm{mml}, 10$ equiv) and PPTS ( $0.05 \mathrm{~g}, 0.38 \mathrm{mmol}, 0.1$ equiv) were added and the resulting mixture was stirred at $\mathrm{rt}$ for $3 \mathrm{~h}$. After completion, saturated aqueous $\mathrm{NaHCO}_{3}$ solution $(2 \mathrm{~mL})$ was added, the organic layer was separated and the aqueous layer was extracted with $\mathrm{CH}_{2} \mathrm{Cl}_{2}(3 \times 20 \mathrm{~mL})$. The combined organic layers were dried over anhydrous $\mathrm{MgSO}_{4}$, filtered and concentrated in vacuo. The residue was purified by flash chromatography (PE/AcOEt : 95/5) to afford the pure acetonide 5 (1.12 g, 96\%).

${ }^{1} \mathrm{H}$ NMR (400 MHZ, $\left.\mathrm{CDCl}_{3}\right): \delta 7.33(\mathrm{~m}, 5 \mathrm{H}), 5.83(\mathrm{~m}, 1 \mathrm{H}), 5.04(\mathrm{~m}, 2 \mathrm{H}), 4.50(\mathrm{~s}, 2 \mathrm{H}), 4.13$ $(\mathrm{m}, 1 \mathrm{H}), 3.57(\mathrm{~m}, 2 \mathrm{H}), 2.20(\mathrm{~m}, 2 \mathrm{H}), 1.76(\mathrm{~m}, 2 \mathrm{H}), 1.46-1.28(\mathrm{~m}+3 \mathrm{~s}, 2+9=11 \mathrm{H})$.

${ }^{13} \mathrm{C}$ NMR (125 MHz, $\left.\mathrm{CDCl}_{3}\right): \delta 138.5(\mathrm{~s}), 134.2(\mathrm{~d}), 128.4(\mathrm{~d}), 127.7,127.6(3 \mathrm{~d}), 117.7(\mathrm{t})$, $98.4(\mathrm{~s}), 73.1(\mathrm{~s}), 72.6(\mathrm{~s}), 66.2(\mathrm{t}), 62.5(\mathrm{~d}), 50.0(\mathrm{t}), 39.2(\mathrm{t}), 36.6(\mathrm{t}), 31.2(\mathrm{q}), 26.4(\mathrm{q}), 25.0$ (q)

GC-MS (EI) $m / z 304\left(\mathrm{M}^{+}\right), 289,263,145,91$

IR (film) v 2920, $1096 \mathrm{~cm}^{-1}$

$[\alpha]_{20}^{\mathrm{D}}=-2.38^{\circ}\left(c=0.15, \mathrm{CHCl}_{3}\right)$

1-[6-(2-Benzyloxy-ethyl)-2,2,4-trimethyl-[1,3]dioxan-4-yl]-pent-4-en-2-ol (6)

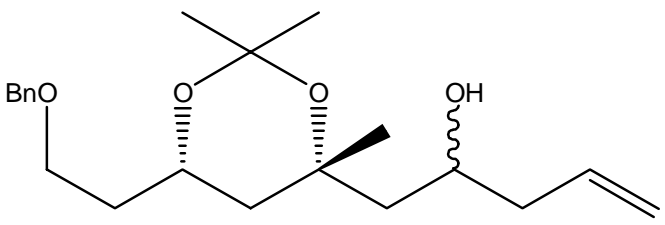

To a solution of $5\left(0.2 \mathrm{~g}, 0.65 \mathrm{mmol}, 1\right.$ equiv) in a 3:1 dioxane $/ \mathrm{H}_{2} \mathrm{O}$ mixture $(5 \mathrm{~mL}: 1.6 \mathrm{~mL})$ at $\mathrm{rt}$ was successively added $\mathrm{OsO}_{4}(0.013 \mathrm{mmol}, 0.02$ equiv $), 2,6$-lutidine $(0.15 \mathrm{~mL}$, $1.3 \mathrm{mmol}, 2$ equiv) and $\mathrm{NaIO}_{4}(0.555 \mathrm{~g}, 2.6 \mathrm{mmol}, 4$ equiv). The solution was stirred at this temperature until completion $(c a .3 \mathrm{~h})$ then quenched by addition of water $(2 \mathrm{~mL})$ followed by $\mathrm{CH}_{2} \mathrm{Cl}_{2}(5 \mathrm{~mL})$. The aqueous layer was extracted with $\mathrm{CH}_{2} \mathrm{Cl}_{2}(3 \times 10 \mathrm{~mL})$, the combined organic layers were dried over anhydrous $\mathrm{MgSO}_{4}$, filtered and concentrated in vacuo. The residue was not purified and used as it in the next step. 
Crude aldehyde was dissolved in freshly distilled $\mathrm{Et}_{2} \mathrm{O}(1.5 \mathrm{~mL})$ and the solution was cooled to $-78{ }^{\circ} \mathrm{C}$. Allymagnesium bromide $2.0 \mathrm{M}$ solution $(0.65 \mathrm{~mL}, 1.3 \mathrm{mmol}, 2$ equiv) was added dropwise and the mixture was allowed to stir at $-78{ }^{\circ} \mathrm{C}$ for $2 \mathrm{~h}$. Then saturated aqueous $\mathrm{NH}_{4} \mathrm{Cl}$ solution $(5 \mathrm{~mL})$ was added, the mixture was extracted with $\mathrm{CH}_{2} \mathrm{Cl}_{2}(3 \times 20 \mathrm{~mL})$ and the combined organic layers were dried over anhydrous $\mathrm{MgSO}_{4}$, filtered and concentrated in vacuo. The residue was purified by flash chromatography (PE/AcOEt : 8/2) to afford 6 as a 1:1 mixture of diastereomers (185 mg, 82\%).

${ }^{1} \mathrm{H}$ NMR (400 MHZ, $\left.\mathrm{CDCl}_{3}\right): \delta 7.33(\mathrm{~m}, 5 \mathrm{H}), 5.83(\mathrm{~m}, 1 \mathrm{H}), 5.07(\mathrm{~m}, 2 \mathrm{H}), 4.51(\mathrm{~s}, 2 \mathrm{H}), 4.27$ (bs, $0.5 \mathrm{H}), 4.21(\mathrm{~m}, 1 \mathrm{H}), 4.13(\mathrm{bs}, 0.5 \mathrm{H}), 4.06(\mathrm{~m}, 1 \mathrm{H}), 3.58(\mathrm{~m}, 2 \mathrm{H}), 2.20(\mathrm{~m}, 2 \mathrm{H}), 1.76(\mathrm{~m}$, $3 \mathrm{H}), 1.61(\mathrm{~m}, 1 \mathrm{H}), 1.50-1.15(\mathrm{~m}, 11 \mathrm{H})$.

${ }^{13} \mathrm{C}$ NMR (125 MHz, $\left.\mathrm{CDCl}_{3}\right): \delta 138.5(\mathrm{~s}), 135.1(\mathrm{~d}), 128.4,127.7,127.6(3 \mathrm{~d}), 117.0,116.9$ (2t), 99.0, $98.7(2 \mathrm{~s}), 76.7(\mathrm{t}), 75.1,74.9(2 \mathrm{~s}), 73.1(\mathrm{t}), 68.1,67.3(2 \mathrm{~d}), 66.1,66.0(2 \mathrm{t}), 62.5$, $62.3(2 \mathrm{~d}), 51.5,49.2(2 \mathrm{t}), 42.3,42.0(2 \mathrm{t}), 37.4,36.4(2 \mathrm{t}), 31.8(\mathrm{q}), 28.7(\mathrm{q}), 25.0,24.7(2 \mathrm{q})$

GC-MS (EI) m/z $347\left(\mathrm{M}-\mathrm{H}^{+}\right), 333,107,91$

IR (film) v 3500, 2989, 2938, 2864, 1378, 1199, $1129 \mathrm{~cm}^{-1}$

\section{\{1-[6-(2-Benzyloxy-ethyl)-2,2,4-trimethyl-[1,3]dioxan-4-ylmethyl]-but-3-enyloxy\}-tert-} butyl-dimethyl-silane (7)

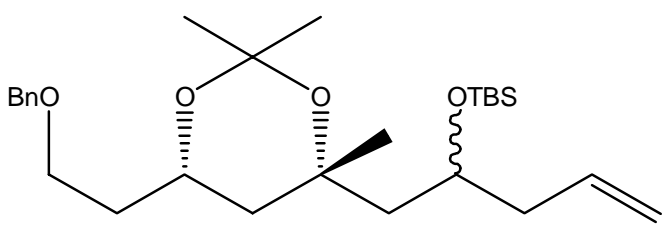

To a solution of $6(1.0 \mathrm{~g}, 2.87 \mathrm{mmol}, 1$ equiv $)$ in $\mathrm{CH}_{2} \mathrm{Cl}_{2}(5 \mathrm{~mL})$ at $-78{ }^{\circ} \mathrm{C}$ was added 2,6-lutidine ( $0.67 \mathrm{~mL}, 5.74 \mathrm{mmol}, 2$ equiv) and TBSOTf ( $0.99 \mathrm{~mL}, 4.3 \mathrm{mmol}, 1.6$ equiv). The solution was stirred at this temperature for $3 \mathrm{~h}$. Saturated aqueous $\mathrm{NH}_{4} \mathrm{Cl}$ solution $(1 \mathrm{~mL})$ was added to the mixture and the organic layer was separated. The aqueous layer was extracted with $\mathrm{CH}_{2} \mathrm{Cl}_{2}(3 \times 20 \mathrm{~mL})$, the combined organic layers were dried over anhydrous $\mathrm{MgSO}_{4}$, filtered and concentrated in vacuo. The residue was purified by flash chromatography (PE/AcOEt : $95 / 5)$ to afford 7 as a 1:1 mixture of diastereomers $(1.15 \mathrm{~g}, 89 \%)$.

${ }^{1} \mathrm{H}$ NMR (400 MHZ, $\left.\mathrm{CDCl}_{3}\right): \delta 7.31(\mathrm{~m}, 5 \mathrm{H}), 5.83(\mathrm{~m}, 1 \mathrm{H}), 5.02(\mathrm{~m}, 2 \mathrm{H}), 4.49(\mathrm{~s}, 2 \mathrm{H}), 4.12$ $(\mathrm{m}, 1 \mathrm{H}), 4.00(\mathrm{~m}, 1 \mathrm{H}), 3.56(\mathrm{~m}, 2 \mathrm{H}), 2.28(\mathrm{~m}, 2 \mathrm{H}), 1.82-1.58(\mathrm{~m}, 15 \mathrm{H}), 0.87(\mathrm{~s}, 9 \mathrm{H}), 0.06(\mathrm{~m}$, $6 \mathrm{H})$.

${ }^{13} \mathrm{C}$ NMR $\left(125 \mathrm{MHz}, \mathrm{CDCl}_{3}\right): \delta 138.5$ (s), 135.5, 135,4 (2d), 128.4, $127.7127 .6(3 \mathrm{~d}), 116.8$, $116.7(2 \mathrm{t}), 98.3,98.2(2 \mathrm{~s}), 73.1(\mathrm{t}), 72.5,72.3(2 \mathrm{~s}), 68.7,68.3(2 \mathrm{~d}), 66.3,66.2(2 \mathrm{t}), 62.5,62.3$ $(2 \mathrm{~d}), 52.8,51.8(2 \mathrm{t}), 43.6,43.3(2 \mathrm{t}), 41.8(\mathrm{t}), 38.9(\mathrm{t}), 36.6(\mathrm{t}), 32.0,31.8(2 \mathrm{q}), 27.8(\mathrm{q}), 26.0$, $26.0(2 \mathrm{q}), 25.1,24.9(2 \mathrm{q}), 18.1(\mathrm{~s}),-3.8,-3.9,-4.2,4.3(4 \mathrm{q})$

GC-MS (EI) $m / z 462\left(\mathrm{M}^{+}\right), 447,263,185,145,91$

IR (film) v 2929, 2856, 1375, 1255, 1196, 1075, 910, 835, $734 \mathrm{~cm}^{-1}$ 


\section{7-[6-(2-Benzyloxy-ethyl)-2,2,4-trimethyl-[1,3]dioxan-4-yl]-6-(tert-butyl-dimethyl-}

silanyloxy)-hept-1-en-4-ol (8)

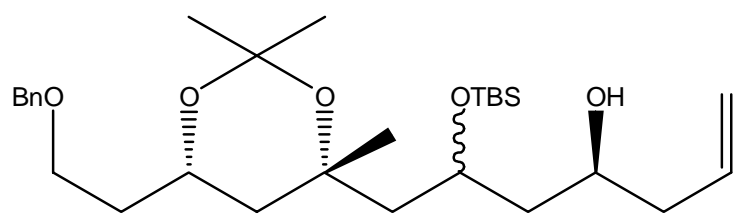

To a solution of $7\left(0.70 \mathrm{~g}, 1.56 \mathrm{mmol}, 1\right.$ equiv) in a 3:1 dioxane/ $\mathrm{H}_{2} \mathrm{O}$ mixture $(12 \mathrm{~mL}: 4 \mathrm{~mL})$ at $\mathrm{rt}$ was successively added $\mathrm{OsO}_{4}(0.03 \mathrm{mmol}, 0.02$ equiv $), 2,6$-lutidine $(0.36 \mathrm{~mL}, 3.1 \mathrm{mmol}$, 2 equiv) and $\mathrm{NaIO}_{4}(1.33 \mathrm{~g}, 6.2 \mathrm{mmol}, 4$ equiv). The solution was stirred at this temperature until completion $(\mathrm{ca} .3 \mathrm{~h})$ then quenched by addition of water $(5 \mathrm{~mL})$ followed by $\mathrm{CH}_{2} \mathrm{Cl}_{2}(10$ $\mathrm{mL})$. The aqueous layer was extracted with $\mathrm{CH}_{2} \mathrm{Cl}_{2}(3 \times 20 \mathrm{~mL})$, the combined organic layers were dried over anhydrous $\mathrm{MgSO}_{4}$, filtered and concentrated in vacuo. The residue was not purified and used without further purification in the next step.

In a flame-dried round-bottom flask under Ar was transferred Ti(S,S)(TADDOL) complex ${ }^{3}$ $\left(1.24 \mathrm{~g}, 2.0 \mathrm{mmol}, 1.3\right.$ equiv) in freshly distilled $\mathrm{Et}_{2} \mathrm{O}(10 \mathrm{~mL})$. The mixture was cooled to $-78^{\circ} \mathrm{C}$ and allylmagnesium bromide $2.0 \mathrm{M}(0.86 \mathrm{~mL}, 1.7 \mathrm{mmol}, 1.08$ equiv) was added dropwise. The mixture was allowed to stirr at $0{ }^{\circ} \mathrm{C}$ for $2 \mathrm{~h}$. After cooling to $-78^{\circ} \mathrm{C}$, a solution of the latter aldehyde in $\mathrm{Et}_{2} \mathrm{O}(10 \mathrm{~mL})$ was added dropwise. The mixture was stirred for $1 \mathrm{~h}$ and then water $(20 \mathrm{~mL})$ was added. The resulting suspension was stirred overnight. The mixture was then extracted with $\mathrm{Et}_{2} \mathrm{O}(3 \mathrm{x} 50 \mathrm{~mL})$, the combined organic layers were dried over anhydrous $\mathrm{MgSO}_{4}$, filtered and concentrated in vacuo. The residue was purified by flash chromatography (PE/AcOEt : 9/1) to afford 8 as a $1: 1$ mixture of diastereomers $(0.738 \mathrm{~g}$, $96 \%)$.

${ }^{1} \mathrm{H}$ NMR (400 MHZ, $\left.\mathrm{CDCl}_{3}\right): \delta 7.32(\mathrm{~m}, 5 \mathrm{H}), 5.83(\mathrm{~m}, 1 \mathrm{H}), 5.06(\mathrm{~m}, 2 \mathrm{H}), 4.49(\mathrm{~s}, 2 \mathrm{H}), 4.20-$ $3.70(\mathrm{~m}, 3 \mathrm{H}), 3.56(\mathrm{~m}, 2 \mathrm{H}), 2.21(\mathrm{~m}, 2 \mathrm{H}), 1.92-1.50(\mathrm{~m}, 6 \mathrm{H}), 1.45-1.10(\mathrm{~m}, 12 \mathrm{H}), 0.87(\mathrm{~s}$, 9H), 0.09 (m, 6H).

${ }^{13} \mathrm{C}$ NMR (125 MHz, $\left.\mathrm{CDCl}_{3}\right): \delta 138.5$ (s), 135.3 (d), $135.2(\mathrm{~d}), 128.4,127.7,127.6$ (3d), $117.0(\mathrm{t}), 98.7,98,6,98.5(3 \mathrm{~s}), 73.1(\mathrm{t}), 72.6,72.5(2 \mathrm{~s}), 68.7,68.6,68.3(3 \mathrm{~d}), 66.1,66.0(2 \mathrm{t})$, 62.4, $62.3(2 \mathrm{~d}), 53.5,51.1(2 \mathrm{t}), 44.3,43.1,42.4(3 \mathrm{t}), 41.8,39.0,36.5,36.4(4 \mathrm{t}), 31.8,31.7$ (2q), $27.8(q), 25.9,25.8,25.6,24.9,24.8$ (6q), $17.9(\mathrm{~s}),-4.0,-4.1,-4.2,-4.3,-4.4(5 q)$

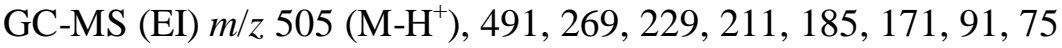

HRMS calcd for $\mathrm{C}_{29} \mathrm{H}_{51} \mathrm{O}_{5} \mathrm{Si}[\mathrm{M}+\mathrm{H}]^{+}:$507.3506; found, 507.3513

IR (film) v 3301, 2931, 2245, 2857, 1446, 1371, 907, $728 \mathrm{~cm}^{-1}$

9-[6-(2-Benzyloxy-ethyl)-2,2,4-trimethyl-[1,3]dioxan-4-yl]-8-(tert-butyl-dimethylsilanyloxy)-non-1-ene-4,6-diol (10)

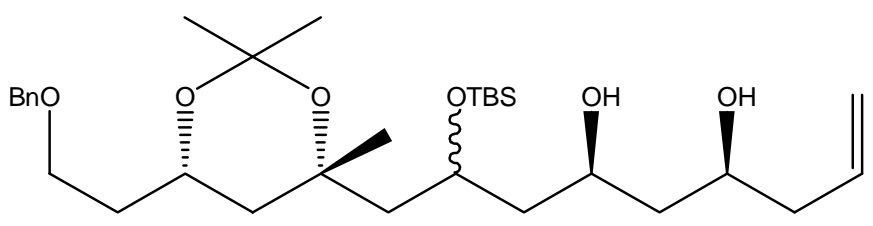


To a solution of $8\left(0.739 \mathrm{~g}, 1.5 \mathrm{mmol}, 1\right.$ equiv) in a $3: 1$ dioxane/ $\mathrm{H}_{2} \mathrm{O}$ mixture $(12 \mathrm{~mL}: 4 \mathrm{~mL})$ at $\mathrm{rt}$ was successively added $\mathrm{OsO}_{4}(0.03 \mathrm{mmol}, 0.02$ equiv), 2,6-lutidine $(0.35 \mathrm{~mL}, 3 \mathrm{mmol}, 2$ equiv) and $\mathrm{NaIO}_{4}(1.28 \mathrm{~g}, 6 \mathrm{mmol}, 4$ equiv). The solution was stirred at this temperature until completion $(c a .3 \mathrm{~h})$ then quenched by addition of water $(5 \mathrm{~mL})$ followed by $\mathrm{CH}_{2} \mathrm{Cl}_{2}(10 \mathrm{~mL})$. The aqueous layer was extracted with $\mathrm{CH}_{2} \mathrm{Cl}_{2}(3 \times 20 \mathrm{~mL})$, the combined organic layers were dried over anhydrous $\mathrm{MgSO}_{4}$, filtered and concentrated in vacuo. The residue was not purified and used without further purification in the next step.

To a flame-dried round-bottom flask under $\mathrm{Ar}$ was added $\mathrm{CH}_{2} \mathrm{Cl}_{2}(4.5 \mathrm{~mL}), \mathrm{SnCl}_{4}(0.21 \mathrm{~mL}$, $1.8 \mathrm{mmol}, 1$ equiv) and allyltrimethylsilane $(0.29 \mathrm{~mL}, 1.8 \mathrm{mmol}, 1$ equiv) and the solution was stirred at $\mathrm{rt}$ for $12 \mathrm{~h}$. This solution was transferred by a canula to a round-bottom flask containing a solution of the latter crude aldehyde in $\mathrm{CH}_{2} \mathrm{Cl}_{2}(10 \mathrm{~mL})$ at $-78{ }^{\circ} \mathrm{C}$. The mixture was allowed to stir at this temperature for $1 \mathrm{~h}$. Work-up entailed addition of $\mathrm{MeOH}(2 \mathrm{~mL})$, saturated aqueous $\mathrm{NH}_{4} \mathrm{Cl}$ solution $(4 \mathrm{~mL})$ and extraction of the resulting mixture with $\mathrm{CH}_{2} \mathrm{Cl}_{2}$ ( $3 \times 15 \mathrm{~mL}$ ). The combined organic layers were dried over anhydrous $\mathrm{MgSO}_{4}$, filtered and concentrated in vacuo. The residue was purified by flash chromatography (PE/AcOEt : 8/2) to afford $\mathbf{1 0}$ as a 1:1 mixture of diastereomers $(0.752 \mathrm{~g}, 91 \%)$.

${ }^{1} \mathrm{H}$ NMR (400 MHZ, $\left.\mathrm{CDCl}_{3}\right): \delta 7.31(\mathrm{~m}, 5 \mathrm{H}), 5.83(\mathrm{~m}, 1 \mathrm{H}), 5.06(\mathrm{~m}, 2 \mathrm{H}), 4.49(\mathrm{~s}, 2 \mathrm{H}), 4.32$ $(\mathrm{m}, 1 \mathrm{H}), 4.22(\mathrm{~m}, 2 \mathrm{H}), 4.13(\mathrm{~m}, 2 \mathrm{H}), 4.04(\mathrm{~m}, 1 \mathrm{H}), 3.91(\mathrm{~m}, 1 \mathrm{H}), 3.56(\mathrm{~m}, 2 \mathrm{H}), 2.22(\mathrm{~m}, 2 \mathrm{H})$, $1.96(\mathrm{~m}, 1 \mathrm{H}), 1.83-1.12(\mathrm{~m}, 20 \mathrm{H}), 0.80(\mathrm{~s}, 9 \mathrm{H}), 0.06(\mathrm{~m}, 6 \mathrm{H})$.

${ }^{13} \mathrm{C}$ NMR (125 MHz, $\left.\mathrm{CDCl}_{3}\right): \delta 138.5$ (s), 135.2, 135.1, 130.9, 128.4, 127.7, 127.6 (6d), 117.1, $117.0(2 \mathrm{t}), 98.9,98.6(2 \mathrm{~s}), 73.1(\mathrm{t}), 73.0,72.7(2 \mathrm{~s}), 71.7,71.6(2 \mathrm{~d}), 70.3,70.0,68.9$, $67.3(4 \mathrm{~d}), 66.1,66.0(2 \mathrm{t}), 62.4,62.3(2 \mathrm{~d}), 53.2,50.7(2 \mathrm{t}), 45.5,43.8,43.0,42.2(4 \mathrm{t}), 41.9$, $38.6,36.5,36.4(4 \mathrm{t}), 31.7,28.0(2 \mathrm{q}), 25.8,25.3,24.8(3 \mathrm{q}), 17.9(\mathrm{~s}),-4.2,-4.4,-4.5(3 \mathrm{q})$

GC-MS (EI) $m / z 549\left(\mathrm{M}-\mathrm{H}^{+}\right), 535,229,211,171,91,75$

HRMS calcd for $\mathrm{C}_{31} \mathrm{H}_{55} \mathrm{O}_{6} \mathrm{Si}[\mathrm{M}+\mathrm{H}]^{+}:$551.3768; found, 551.3762

IR (film) v 3302, 2990, 2937, 2857, 1378, 1198, 1159, 1120, $825 \mathrm{~cm}^{-1}$

\{1-(6-Allyl-2,2-dimethyl-[1,3]dioxan-4-ylmethyl)-2-[6-(2-benzyloxy-ethyl)-2,2,4trimethyl-[1,3]dioxan-4-yl]-ethoxy\}-tert-butyl-dimethyl-silane (11)

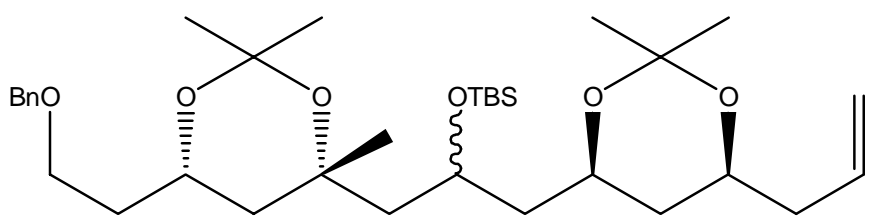

Compound 10 (0.826 g, $1.5 \mathrm{mmol}, 1$ equiv) was dissolved in $\mathrm{CH}_{2} \mathrm{Cl}_{2}$ (3 mL) at rt. 2,2dimethoxypropane (1.8 mL, $15 \mathrm{mmol}, 10$ equiv) and PPTS (0.038 g, $0.15 \mathrm{mmol}, 0.1$ equiv) were added and the resulting mixture was stirred at room temperature for $3 \mathrm{hs}$. After completion, saturated aqueous $\mathrm{NaHCO}_{3}$ solution $(3 \mathrm{~mL})$ was added, the organic layer was separated and the aqueous layer was extracted with $\mathrm{CH}_{2} \mathrm{Cl}_{2}(3 \times 20 \mathrm{~mL})$. The combined organic layers were dried over anhydrous $\mathrm{MgSO}_{4}$, filtered and concentrated in vacuo. The residue was purified by flash chromatography (PE/AcOEt : 95/5) to afford acetonide 11 (0.860 g, $97 \%$ ). 
${ }^{1} \mathrm{H}$ NMR (400 MHZ, $\left.\mathrm{CDCl}_{3}\right): \delta 7.31(\mathrm{~m}, 5 \mathrm{H}), 5.80(\mathrm{~m}, 1 \mathrm{H}), 5.05(\mathrm{~m}, 2 \mathrm{H}), 4.51(\mathrm{~s}, 2 \mathrm{H}), 4.10-$ $3.80(\mathrm{~m}, 3 \mathrm{H}), 3.58(\mathrm{~m}, 2 \mathrm{H}), 2.17(\mathrm{~m}, 2 \mathrm{H}), 1.88-1.00(\mathrm{~m}, 26 \mathrm{H}), 0.87(\mathrm{~s}, 9 \mathrm{H}), 0.06(\mathrm{~m}, 6 \mathrm{H})$.

${ }^{13} \mathrm{C} \mathrm{NMR}\left(125 \mathrm{MHz}, \mathrm{CDCl}_{3}\right): \delta 138.5(\mathrm{~s}), 134.4,134.2,128.4,127.7,127.6(5 \mathrm{~d}), 117.0,116.9$ $(2 \mathrm{t}), 98.4,98.3,98.3(3 \mathrm{~s}), 73.1(\mathrm{t}), 72.4(\mathrm{~s}), 68.7,68.6(2 \mathrm{~d}), 66.3,66.3(2 \mathrm{t}), 65.9,65.4(2 \mathrm{~d})$, $62.4(\mathrm{~d}), 53.4(\mathrm{t}), 46.9,40.9,40.9,39.4,37.2,36.9,36.6(7 \mathrm{t}), 30.3,30.2,27.5,26.0,25.9$, $25.8,25.1,25.0,20.2,19.9(10 \mathrm{q}), 18.0,17.9(2 \mathrm{~s}), 14.2(\mathrm{q}),-3.8,-4.0,-4.1,-4.2(4 \mathrm{q})$

GC-MS (EI) $m / z, 575\left({\mathrm{M}-\mathrm{CH}_{3}}^{+}\right), 353,281,207,155,107,91,75$

HRMS calcd for $\mathrm{C}_{34} \mathrm{H}_{58} \mathrm{NO}_{6} \mathrm{Si}\left[\mathrm{M}+\mathrm{NH}_{3}\right]^{+}:$608.4302; found, 608.4342

IR (film) v 2990, 2936, 2857, 1378, 1197, 1169, 1099, $835 \mathrm{~cm}^{-1}$

\section{1-(6-Allyl-2,2-dimethyl-[1,3]dioxan-4-yl)-3-[6-(2-benzyloxy-ethyl)-2,2,4-trimethyl- $[1,3]$ dioxan-4-yl]-propan-2-ol (12)}

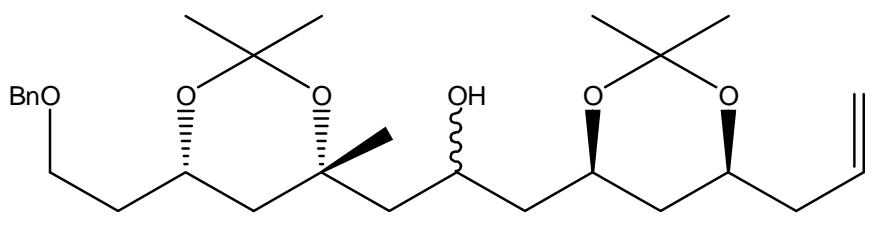

To a solution of $11(0.591 \mathrm{~g}, 1 \mathrm{mmol}, 1$ equiv) in THF $(5 \mathrm{~mL})$ at $\mathrm{rt}$ was added TBAF $1.0 \mathrm{M}$ ( $4 \mathrm{~mL}, 4 \mathrm{mmol}, 4$ equiv). The solution was stirred for $16 \mathrm{~h}$. Saturated aqueous $\mathrm{NH}_{4} \mathrm{Cl}$ solution $(5 \mathrm{~mL})$ was added and the resulting mixture was extracted with $\mathrm{CH}_{2} \mathrm{Cl}_{2}(3 \times 20 \mathrm{~mL})$. The combined organic layers were dried over anhydrous $\mathrm{MgSO}_{4}$, filtered and concentrated in vacuo. The residue was purified by flash chromatography (PE/AcOEt : 8/2) to afford 12 as a 1:1 mixture of diastereomers $(0.438 \mathrm{~g}, 92 \%)$.

${ }^{1} \mathrm{H}$ NMR (400 MHZ, $\left.\mathrm{CDCl}_{3}\right): \delta 7.31(\mathrm{~m}, 5 \mathrm{H}), 5.80(\mathrm{~m}, 1 \mathrm{H}), 5.08(\mathrm{~m}, 2 \mathrm{H}), 4.50(\mathrm{~s}, 2 \mathrm{H}), 4.16$ $(\mathrm{m}, 3 \mathrm{H}), 3.89(\mathrm{~m}, 1 \mathrm{H}), 3.56(\mathrm{~m}, 2 \mathrm{H}), 2.22(\mathrm{~m}, 2 \mathrm{H}), 1.85-1.58(\mathrm{~m}, 5 \mathrm{H}), 1.50-1.30(\mathrm{~m}, 19 \mathrm{H})$, $1.19(\mathrm{~m}, 2 \mathrm{H})$.

${ }^{13} \mathrm{C}$ NMR (125 MHz, $\left.\mathrm{CDCl}_{3}\right): \delta 138.5(\mathrm{~s}), 134.3(\mathrm{~d}), 128.4,127.7,127.6(3 \mathrm{~d}), 117.1,117.0$ (2t), 99.0, 98.67, 98.6, 98.5 (4s), 75.0, $74.9(2 \mathrm{~s}), 73.1(\mathrm{t}), 68.7,68.7(2 \mathrm{~d}), 66.6(\mathrm{~d}), 66.0,65.9$ $(\mathrm{t}), 65.8(\mathrm{~d}), 64.8,64.7(2 \mathrm{~d}), 62.5,62.4(2 \mathrm{~d}), 52.7(\mathrm{t}), 50.4(\mathrm{t}), 45.0(\mathrm{t}), 43.8(\mathrm{t}), 41.8(\mathrm{t}), 40.8$ $(\mathrm{t}), 38.0(\mathrm{t}), 37.1(\mathrm{t}), 36.5,36.4,36.2(3 \mathrm{t}), 31.8(\mathrm{q}), 31.7(\mathrm{q}), 30.3(\mathrm{q}), 28.3(\mathrm{q}), 25.2(\mathrm{q}), 24.7$, $24.6(2 \mathrm{q}), 19.9(\mathrm{q})$.

GC-MS (EI) $m / z 475\left(\mathrm{M}-\mathrm{H}^{+}\right), 461,281,207,91$

HRMS calcd for $\mathrm{C}_{28} \mathrm{H}_{45} \mathrm{O}_{6}[\mathrm{M}+\mathrm{H}]^{+}$: 477.3216; found, 477.3223

IR (film) v 3508, 2990, 2941, 2863, 1379, 1199, $1124 \mathrm{~cm}^{-1}$ 
1-(6-Allyl-2,2-dimethyl-[1,3]dioxan-4-yl)-3-[6-(2-benzyloxy-ethyl)-2,2,4-trimethyl[1,3]dioxan-4-yl]-propan-2-one (13)

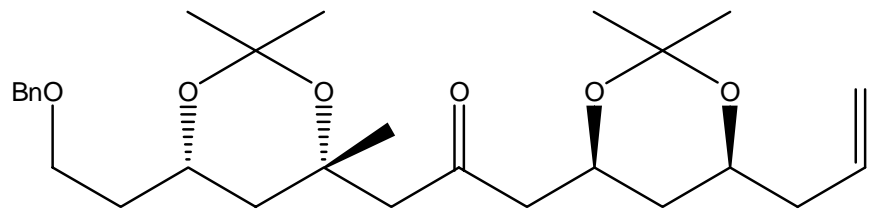

To a $0.1 \mathrm{M}$ solution of alcohol $12\left(0.238 \mathrm{~g}, 0.5 \mathrm{mmol}\right.$, 1 equiv) in a $1: 1 \mathrm{CH}_{2} \mathrm{Cl}_{2}$ :DMSO mixture $(2.5 \mathrm{~mL}: 2.5 \mathrm{~mL})$ at $0^{\circ} \mathrm{C}$ were added $\mathrm{Et}_{3} \mathrm{~N}\left(0.70 \mathrm{~mL}, 5 \mathrm{mmol}, 10\right.$ equiv) and $\mathrm{Pyr} \mathrm{SO}_{3}$ complex $(0.796 \mathrm{~g}, 5 \mathrm{mmol}, 10$ equiv). After $72 \mathrm{~h}$, the mixture was quenched with water $(2 \mathrm{~mL})$ and extracted with $\mathrm{CH}_{2} \mathrm{Cl}_{2}(3 \times 10 \mathrm{~mL})$. The combined organic layers were dried over anhydrous $\mathrm{MgSO}_{4}$, filtered and concentrated in vacuo. The residue was purified by flash chromatography (PE/AcOEt : 8/2) to afford the pure ketone $\mathbf{1 3}$ as a single diastereomer $(0.209 \mathrm{~g}, 88 \%,>98 e e)$.

${ }^{1} \mathrm{H}$ NMR (400 MHZ, $\left.\mathrm{CDCl}_{3}\right): \delta 7.32(\mathrm{~m}, 5 \mathrm{H}), 5.87(\mathrm{~m}, 1 \mathrm{H}), 5.08(\mathrm{~m}, 2 \mathrm{H}), 4.50(\mathrm{~s}, 2 \mathrm{H}), 4.32$ $(\mathrm{m}, 1 \mathrm{H}), 4.13(\mathrm{~m}, 1 \mathrm{H}), 3.89(\mathrm{~m}, 1 \mathrm{H}), 3.56(\mathrm{~m}, 2 \mathrm{H}), 2.73(\mathrm{~m}, 1 \mathrm{H}), 2.53(\mathrm{~m}, 2 \mathrm{H}), 2.24(\mathrm{~m}, 2 \mathrm{H})$, $1.74(\mathrm{~m}, 2 \mathrm{H}), 1.72-1.47(\mathrm{~m}, 3 \mathrm{H}), 1.44(\mathrm{~s}, 3 \mathrm{H}), 1.42(\mathrm{~s}, 3 \mathrm{H}), 1.39(\mathrm{~s}, 3 \mathrm{H}), 1.35(\mathrm{~s}, 3 \mathrm{H}), 1.34$ (s, $3 \mathrm{H}), 1.11(\mathrm{~m}, 2 \mathrm{H})$.

${ }^{13} \mathrm{C} \mathrm{NMR}\left(125 \mathrm{MHz}, \mathrm{CDCl}_{3}\right): \delta 207.8(\mathrm{~s}), 137.5(\mathrm{~s}), 134.1(\mathrm{~d}), 128.4,127.7,127.6(3 \mathrm{~d}), 117.1$ $(\mathrm{t}), 97.6(\mathrm{~s}), 73.1(\mathrm{t}), 72.8(\mathrm{~s}), 68.5(\mathrm{~d}), 66.1(\mathrm{t}), 65.5(\mathrm{~d}), 62.3(\mathrm{~d}), 58.0(\mathrm{t}), 51.5(\mathrm{t}), 40.8(\mathrm{t})$, $39.6(\mathrm{t}), 36.4(\mathrm{t}), 36.3(\mathrm{t}), 31.7(\mathrm{q}), 30.1(\mathrm{q}), 26.5(\mathrm{q}), 24.9(\mathrm{q}), 19.8(\mathrm{q})$

GC-MS (EI) $m / z 474\left(\mathrm{M}^{+}\right), 459,281,207,91$

HRMS calcd for $\mathrm{C}_{28} \mathrm{H}_{46} \mathrm{NO}_{6}\left[\mathrm{M}+\mathrm{NH}_{4}\right]^{+}$: 492.3325; found, 492.3330

IR (film) v 2991, 2927, 1720, 1379, $1198 \mathrm{~cm}^{-1}$

$[\alpha]_{20}^{\mathrm{D}}=-3.3^{\circ}\left(c=0.50, \mathrm{CHCl}_{3}\right)$

8-Allyl-2-(2-benzyloxy-ethyl)-4-methyl-1,7-dioxa-spiro[5.5] undecane-4,10-diol (AB spiroketal) (14)

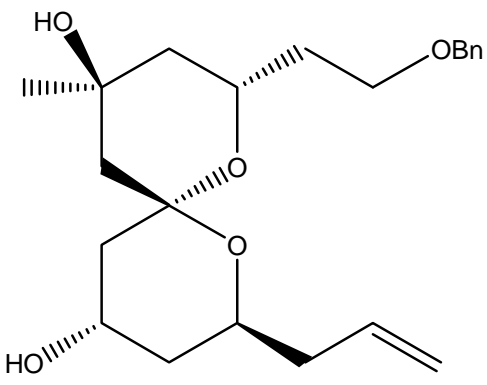

To a solution of $13\left(0.150 \mathrm{~g}, 0.4 \mathrm{mmol}, 1\right.$ equiv) in a 1:1 $\mathrm{CH}_{2} \mathrm{Cl}_{2}: \mathrm{MeOH}$ mixture $(0.5 \mathrm{~mL}: 0.5$ $\mathrm{mL})$ at $\mathrm{rt}$ was added PPTS ( $0.010 \mathrm{~g}, 0.04 \mathrm{mmol}, 0.1$ equiv). The solution was stirred at this temperature for $24 \mathrm{~h}$. One drop of $\mathrm{Et}_{3} \mathrm{~N}$ was added and the mixture was concentrated in vacuo. 
The residue was purified by flash chromatography (PE/AcOEt : 8/2) to afford the $\mathrm{AB}$ spiroketal $14(0.095 \mathrm{~g}, 63 \%,>98 \% e e)$ and the hemiacetal $(20 \%)$. The latter was submitted to the same reaction conditions and provided an extra $10 \%$ of 14.

${ }^{1} \mathrm{H}$ NMR (400 MHZ, $\left.\mathrm{CDCl}_{3}\right): \delta 7.31(\mathrm{~m}, 5 \mathrm{H}), 5.61(\mathrm{~m}, 1 \mathrm{H}), 5.07(\mathrm{~m}, 2 \mathrm{H}), 4.51(\mathrm{~s}, 2 \mathrm{H}), 4.41$ $(\mathrm{s}, 1 \mathrm{H}), 4.16(\mathrm{~m}, 1 \mathrm{H}), 4.01(\mathrm{~m}, 3 \mathrm{H}), 3.58(\mathrm{~m}, 2 \mathrm{H}), 2.11(\mathrm{~m}, 2 \mathrm{H}), 1.88-1.25(\mathrm{~m}, 10 \mathrm{H}), 1.15(\mathrm{~s}$, $3 \mathrm{H})$.

${ }^{13} \mathrm{C}$ NMR (125 MHz, $\left.\mathrm{CDCl}_{3}\right): \delta 138.0(\mathrm{~s}), 134.7(\mathrm{~d}), 128.3,128.1,127.7(3 \mathrm{~d}), 117.9(\mathrm{t}), 100.0$ $(\mathrm{s}), 73.4(\mathrm{t}), 67.8(\mathrm{~s}), 67.3(\mathrm{t}), 64.7(\mathrm{~d}), 64.6(\mathrm{~d}), 64.3(\mathrm{~d}), 45.2(\mathrm{t}), 43.9(\mathrm{t}), 40.3(\mathrm{t}), 39.8(\mathrm{t})$, $37.8(\mathrm{t}), 35.4(\mathrm{t}), 29.9(\mathrm{q})$

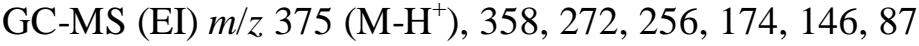

HRMS calcd for $\mathrm{C}_{22} \mathrm{H}_{33} \mathrm{O}_{5}[\mathrm{M}+\mathrm{H}]^{+}: 377.2328$; found, 377.2321

IR (film) v 3511, 2927, 1404, 1199, 1163, $1090 \mathrm{~cm}^{-1}$

$[\alpha]_{20}^{\mathrm{D}}=-49.8^{\circ}\left(c=0.50, \mathrm{CHCl}_{3}\right)$

References:

1. Liu, Y.-J.; Tropp, B. E.; Engel, R. Can. J. Chem. 1993, 71, 206.

2. Kocienski, P. J.; Street, S. D. A.; Yeates, C.; Campbell, S. F. J. Chem. Soc. Perkin Trans 1 1987, 2171

3. $\operatorname{Ti}(S, S)($ TADDOL) was made using the procedure described in: Hafner, A.; Duthaler, R. O.; Marti, R.; Rihs, G.; Rothe-Streit, P.; Schwarzenbach, F. J. Am. Chem. Soc. 1992, 114, 2321. 